\title{
Bursts detected in hard X-rays by IBIS telescope in 2003-2009
}

\section{Ivan Chelovekov*}

Space Research Institute, Moscow, Russia

E-mail: chelovekov@iki.rssi.ru

\section{Sergei Grebenev}

Space Research Institute, Moscow, Russia

E-mail: grebenev@iki.rssi.ru

In our work we use IBIS/INTEGRAL telescope archival data in order to search for type I X-ray bursts hard 15-25 keV tails. Due to the huge Field Of View (FOV) of the telescope we were able to discover two new X-ray bursters and observe 832 bursts from known bursters, 234 of which were simultaneously observed by the JEM-X/INTEGRAL telescope. Among these 834 bursts 587 were recorded from the well-known burster GX 354-0. We have determined upper limits on the distances to 16 of the observed burst sources by assuming that the Eddington luminosity limit was reached at the brightness maximum of the brightest bursts.

The Extreme and Variable High Energy Sky

September 19-23, 2011

Chia Laguna (Cagliari), Italy

\footnotetext{
* Speaker.
} 


\section{INTRODUCTION}

Type I X-ray bursts (Lewin et al. 1993) are thermonuclear explosions on the surfaces of weakly magnetized neutron stars in binary systems. These events as well as type II X-ray bursts (flares emerging from instabilities of the accretion flow) are the main candidates to explain the vest majority of X-ray flares observed by orbital astrophysical observatories. While type II bursts may generally have arbitrary profiles, type I bursts light curves have quick rise and exponential decay and they start earlier and last longer in softer energy band then in harder. Sources emitting type I $\mathrm{X}$-ray bursts are known as X-ray bursters. The luminosity of such sources may be quite moderate during the quiescence period but during the burst it may increase by one or two orders of magnitude often reaching the critical Eddington limit. This gives us a unique opportunity to discover new Xray bursters which have low persistent luminosity and can not be confidently detected or identified as such even during long observations by modern X-ray telescopes. Besides that due to their peculiar observational properties type I X-ray bursts carry direct information about the processes near the surface of neutron stars under conditions of super strong gravitational field and pressure, ultrahigh temperatures, and relativistic velocities. Another merit in detection of type-I bursts is that along with the detection of coherent pulsations it serves as one of the most reliable criteria for identifying the nature of the compact object (a neutron star) in X-ray binaries.

The INTEGRAL orbital observatory is equipped with unique wide field telescopes that allow sky fields with an area of $\sim 1000$ square degrees to be simultaneously studied with a flux sensitivity better than $1 \mathrm{mCrab}$ (over several hours of observations) and an angular resolution reaching several arc minutes. In addition it dedicates up to $85 \%$ of the observational time to continuous monitoring of of the Galactic center and plane regions, where the bulk of the Galactic stellar mass is concentrated. Therefore, INTEGRAL is best suited for accomplishing such a task.

In our quest to find X-ray bursts we analyzed the time histories of the total count rate from the ISGRI detector of the IBIS telescope on board the INTEGRAL orbital observatory in the 15$25 \mathrm{keV}$ energy range based on observations during the first seven years of its in-orbit operation (February 2003 - April 2009). We attempted to localize all the detected bursts and identify them with persistent X-ray sources within the FOV of the telescope. We compiled a catalog of identified bursts and constructed their time histories in a softer X-ray energy range using data from the JEM-X monitor on board the INTEGRAL observatory if this was permitted by the observational conditions. The best expectations were to detect hitherto unknown bursters or short lived X-ray transients.

The first part of the work containing some results of our search for bursts in the 2003-2004 data was published previously (Chelovekov et al. 2006), as were its extensions (Chelovekov et al. 2007; Chelovekov and Grebenev 2010b). An improvement in the data processing and analysis methods revealed additional weak bursts recorded in this period.

\section{OBSERVATIONS AND DATA ANALYSIS}

The INTEGRAL international orbital gamma-ray observatory (Winkler et al. 2003) was placed in orbit by a Russian PROTON launcher on October 17, 2002 (Eismont et al. 2003). Out of the four on board instruments we use data from the ISGRI detector of the IBIS gamma-ray telescope (Lebrun et al. 2003) and the JEM-X monitor (Lund et al. 2003). 
The detailed explanation of the data reduction procedure is given in Chelovekov et al. (2006). We use detector light curves (time histories of the ISGRI detector count rate considering all the events regardless of the photon incidence angle) in the 15-25 keV energy range to find burst-like events - burst candidates for further analysis.

Light curves for each individual INTEGRAL observation (corresponding to an individual pointing) were generated with a time resolution of $5 \mathrm{~s}$. For this purpose, we used data from the revision 2 isgri_events.fits files selecting events in the required energy range. Subsequently, we made corrections for the detector "dead" time, took into account the possible interruptions in the telemetry flow from one or several of its modules, and ignored the photons from its "hot" pixels.

An excess of the signal-to-noise ratio $(S-\bar{S}) / N$ above a preset threshold $s_{0}$ in a given time bin of the light curve served as a criterion for the presence of a burst candidate. Since the number of events recorded by the detector in each time bin obeys a Poisson distribution, there is a low, but finite probability $p\left(s_{0}\right)$ of recording a random spike even in the absence of a real burst. To filter out such random spikes we initially set a high threshold $s_{0}=6.0$. It ensures that the probability of recording one random burst with $(S-\bar{S}) / N \gtrsim s_{0}$ in the entire time series under consideration (with $M \sim 3.2 \times 10^{7}$ time bins) does not exceed $p\left(s_{0}\right) \times M \simeq 20 \%$. Since the total count rate of the detector depends on the emission from all sources within the FOV of the telescope, its average value $\bar{S}$ and the noise level $N=\overline{S^{2}}-\bar{S}^{2}$ in our formulas were initially determined independently from each individual pointing. Analysis of the detector light curves showed that the count rate variability was significant even within a single observation in some sessions. Therefore, $\bar{S}$ and $N$ were ultimately determined for a 500-s time interval containing the time bin being analyzed. The resulting selection of bursts was also checked manually.

After performing automatic and manual checks of all the burst candidates we realized that there is a substantial number of events that are real type I X-ray bursts, but have light curve detection statistical significance below the adopted threshold $s_{0}=6.0$. Therefore we also ran a scan catching all the events corresponding to $s_{0}=3.0$ threshold. This step increased the number of burst candidates up to over 50000 (the number is in a good accordance with the predictions of Poisson statistics), but allowed us to find over 380 new bursts. We emphasize that all these bursts came from known bursters, because their detection significance is too low to talk about the observation of events from hitherto unknown sources. Even at such a low threshold one may formally expect the appearance of only $p\left(s_{1}\right) \times M / A \simeq 4$ false events over the entire time of observations, where $\mathrm{A}=\left(29 \mathrm{deg} / 12^{\prime}\right)^{2} \simeq 2.1 \times 10^{4}$ is the number of statistically independent (corresponding to the angular resolution) areas in the IBIS field of view. In reality, however, after integration over the entire burst time its significance, as a rule, turned out to be higher than that determined from an individual bin near the light curve maximum.

We used INTEGRAL OSA (Offline Science Analysis - a standard INTEGRAL pipeline) to reconstruct the images of the sky area inside the IBIS field of view in the $15-25 \mathrm{keV}$ energy range accumulated with the same exposure during and immediately before each of the burst candidates. The comparison of statistical significances of the detection of sources in these images let us reveal and identify the burst source.

We analyzed a total of more than 57000 individual observations performed by the INTEGRAL observatory from February 10, 2003, to April 17, 2009. All these data are now in open access in the INTEGRAL archive. The duration of individual observations was typically $\sim 2.2-3.5 \mathrm{ks}$; the 
total exposure time of all the observations used exceeded $158 \mathrm{Ms}$ (it corresponds to $\sim 5$ years of continuous observations).

\section{RESULTS}

As a result of this work we found 834 type I X-ray bursts. Among them 832 bursts were registered from 22 known bursters, one burst from the new X-ray burster which received a name IGR J17380-3749 (see, e.g., Chelovekov et al. 2006, 2007; Chelovekov and Grebenev 2010a) and one burst from AX J1754.2-2754 (Chelovekov and Grebenev 2007) which was hitherto not regarded as a burster.

The main parameters of the localized bursts and the results of their identification are given in the burst catalog available online at http://hea.iki.rssi.ru/integral/dlc. For each burst, the table gives: the date and time $T_{m}$ of its detection (UT) - the second at which the maximum count rate $S_{m}$ is reached during the event; the burst source name; the burst duration $T_{90}$ - the time interval during which the count rate $S$ exceeded its average preburst value $\bar{S}$ by more than $10 \%$ of its peak value; the effective burst duration $T_{e}$ - the ratio of the total number of counts $\Sigma(S-\bar{S}) T_{b}$ recorded from the source over the entire burst time to the peak count rate $\left(S_{m}-\bar{S}\right)$; and the maximum (peak) flux $F_{m}$ determined for a $1 \mathrm{~s}$ interval. The peak fluxes $F_{m}$ are given in units of the flux from the Crab Nebula ${ }^{1}$. They were recalculated using the light curve (the detector count rate $S-\bar{S}$ ) from the absolute source flux $F_{5}$ in $5 \mathrm{~s}$ including the peak $\left(T_{m}-2 \mathrm{~s} ; T_{m}+3 \mathrm{~s}\right)$ determined from the image, i.e., applying all the required corrections (for the dead time, the observation efficiency in the incomplete coding zone, etc.). Analogous parameters are given for the bursts recorded by the JEM-X monitor simultaneously with the IBIS/ISGRI telescope: the burst duration $T_{90}^{j}$, effective duration $T_{e}^{j}$, and peak flux $F_{m}^{j}$ from JEM-X data. The time of the JEM-X maximum count rate could differ from $T_{m}$. For both instruments, the time of the burst maximum, duration, effective duration, and peak flux were measured using the detector light curves with a resolution (bin length) of $T_{b}=1 \mathrm{~s}$.

A list of the observed X-ray bursters with an indication of the number of bursts recorded from each of them, the total exposure times, and the upper limits on the distances to the corresponding systems is listed in the "Bursters" table which is available online at http://hea.iki.rssi.ru/integral/dlc/bpars. The distances were estimated assuming that the luminosity of the source during the brightest burst reached the Eddington limit and a thermonuclear flash developed in matter with a high helium abundance $(X=0)$. Distances are only given for the systems that were within the JEM-X field of view during at least one of the bursts. This allowed us to obtain the burst spectrum in a wide X-ray energy range to fit a black body model to it and to calculate the $2-100 \mathrm{keV}$ flux within the framework of this model to derive a lower limit for the system's bolometric luminosity. Usage of such an estimate of the bolometric luminosity implies that the listed distances to the sources are only upper limits, although they were formally obtained with an accuracy better than $10 \%$.

We see from the "Bursters" table that more than $70 \%$ of all bursts were observed from one and the same source - the well known X-ray burster GX 354-0. For several other bursters the exposure time of the observations is comparable to or even larger than that of GX 354-0. It is therefore

\footnotetext{
${ }^{1}$ Note that $1 \mathrm{Crab}(15-25 \mathrm{keV})=6.1 \times 10^{-9} \mathrm{ergs}^{-1} \mathrm{~cm}^{-2}$ for a source with a power-law spectrum with a photon index $\alpha=2.1$ or $5.8 \times 10^{-9} \mathrm{ergs}^{-1} \mathrm{~cm}^{-2}$ for a source with a Wien spectrum with a temperature $k T \simeq 2.8 \mathrm{keV}$ typical of bursts with photospheric expansion.
} 
obvious that detection of such a large number of bursts from this source is not the result of a selection effect but most likely suggests that the properties of its emission are especially "favorable" for observations in this energy range during bursts. A detailed analysis of the bursts recorded from the source in 2003-2004 by Chelovekov et al. (2006) showed that their duration in the energy range 15-25 keV was $T_{90} \sim 5-6 \mathrm{~s}$ and the recurrence period was $\tau \sim 4 \mathrm{~h}$. We found no clear correlation between the peak flux, duration, and recurrence period of the bursts from this source.

\section{CONCLUSION}

We used archival data from IBIS/ISGRI telescope on board INTEGRAL in 15-25 keV energy range to find and localize type I X-ray bursts from new and known X-ray bursters. Using detector light curves we found over 50000 events with statistical significance over $3 \sigma$ and were able to localize and identify 834 of them with 2 new and 22 previously known bursters.

We notice that the bursts discussed here were recorded in the hard and relatively narrow spectral range $15-25 \mathrm{keV}$ and, therefore, have fairly peculiar properties. It may well be that even the most active of the observed bursters are also the sources of softer bursts that cannot be seen with the IBIS/ISGRI telescope. Some of the sources from the "Bursters" table from which we observed only single bursts are known as very active bursters in the standard X-ray energy range (Lewin et al. 1993; Grebenev et al. 2000; Emel'yanov et al. 2001; Cornelisse et al., 2003), so the ratio of "hard" and "soft" bursts in them is extremely low. Therefore, the recurrence periods listed in the "Bursters" tables were most likely grossly overestimated. Note that, as follows from this table, the average recurrence period of "hard" bursts from the most active burster GX 354-0, $\tau_{h} \sim 1.5 \mathrm{~d}$, is much longer than the recurrence period $\tau \sim 4 \mathrm{~h}$ found from the distribution of the arrival times of individual events from this burster in 2003-2004 (Chelovekov et al. 2006). Obviously, the burst activity of this burster changed greatly over seven years of its observations.

Our burst search algorithm is mainly optimized for detecting short (up to minutes) He and $\mathrm{H} / \mathrm{He}$ bursts, therefore not all the longer events are present in our catalog. For the next paper of the series we are planning to improve our algorithm and search for longer $\mathrm{H} / \mathrm{He}$ and even $\mathrm{C}$ bursts.

During manual analysis of detector light curves we came across several events that are clearly significant X-ray bursts on the image, but have only 2-3 $\sigma$ detection statistical significance on the light curve. We are currently analyzing approximately 1 million of such events to include a yet more complete set of bursts into the next paper of the series.

Up until now we were using JEM-X data solely as supplementary for IBIS/ISGRI bursts. Currently we are performing the same search for bursts using JEM-X data as the one we did for IBIS/ISGRI. The JEM-X FOV is smaller then the one of IBIS, but its working energy range is more convenient for observing type I X-ray bursts, so we are expecting to find several hundreds of such events. This will allow us to perform a more thorough, comprehensive, and less dependent on the selection effects (the energy range) analysis of the dependence of the burst generation rate on the accretion rate (luminosity) for X-ray bursters and to study the distribution of the number of bursts on their parameters. 


\section{ACKNOWLEDGMENTS}

This work is based on the INTEGRAL observational data provided via the Russian and European Science Data Centers of the observatory. The study was supported by the Program of the Russian President for support of young candidates of science (grant MK-4182.2009.2) and leading scientific schools (grant NSH-5069.2010.2), the "Origin, Structure, and Evolution of Objects in the Universe" Program of the Presidium of the Russian Academy of Sciences, and the Russian Foundation for Basic Research (grant 10-02-01466).

\section{References}

[1] (I.V. Chelovekov and S. A. Grebenev), Pis'ma Astron. Zh. 36, 946 (2010a) [Astron. Lett. 36, 895 (2010a)].

[2] (I.V. Chelovekov and S. A. Grebenev), Proceedings Of Science 115, 126 (2010b).

[3] (I.V. Chelovekov, S. A. Grebenev, and R. A. Sunyaev), Proceedings of the 6th Integral Workshop on The Obscured Universe, Moscow, July 2-7, 2006, Ed. by S. A. Grebenev, C.Winkler, and R. A. Sunyaev, ESA SP-622, 445 (2007).

[4] (I.V. Chelovekov, S. A. Grebenev, and R. A. Sunyaev), Pis'ma Astron. Zh. 32, 508 (2006) [Astron. Lett. 32, 456 (2006)].

[5] (R. Cornelisse, J. J. M. in’t Zand, F. Verbunt, et al.), Astron. Astrophys. 405, 1033 (2003).

[6] (N. A. Eismont, A. V. Ditrikh, G. Janin et al.), Astron. Astrophys. 411, L37 (2003).

[7] (A. N. Emel'yanov, V. A. Aref'ev, E. M. Churazov, et al.), Pis'ma Astron. Zh. 27, 908 (2001) [Astron. Lett. 27, 781 (2001)].

[8] (S. A. Grebenev, A. A. Lutovinov, M. N. Pavlinskii, et al.), Preprint IKI RAN, Pr-2031 (2000).

[9] (F. Lebrun, J. P. Leray, P. Lavocat, et al.), Astron. Astrophys. 411, L141 (2003).

[10] (W. H. G. Lewin, J. van Paradijs, and R. Taam), SpaceSci. Rev. 62, 223 (1993).

[11] (N. Lund, C. Budtz-Jorgensen, N. J. Westergaard, et al.), Astron. Astrophys. 411, L231 (2003).

[12] (C. Winkler, T. J.-L. Courvoisier, G. Di Cocco, et al.), Astron. Astrophys. 411, L1 (2003). 